\title{
Optimal Control of Constrained Cognitive Radio Networks with Dynamic Population Size
}

\author{
Mahdi Lotfinezhad, Ben Liang, Elvino S. Sousa
}

\begin{abstract}
In this paper, we consider the problem of optimal control for throughput utility maximization in cognitive radio networks with dynamic user arrivals and departures. The cognitive radio network considered in this paper consists of a number of heterogeneous sub-networks. These sub-networks may be power-constrained and are required to operate in such a way that the average total interference received on primary channels are kept below given thresholds. We develop a control policy that performs joint admission control and resource scheduling. Through Lyapunov optimization techniques, we show that the proposed policy achieves a utility performance within $O(\delta)$ of optimality for any positive $\delta$. We further show that this arbitrarily closeness to optimality comes at the price of having a delay that is $O\left(\frac{1}{\delta}\right)$ in admitting users. We also propose constant factor approximations of the policy for distributed implementation.
\end{abstract}

\section{INTRODUCTION}

One main driving force behind implementing cognitive radio in wireless networking is the fact that many portions of the spectrum are used only sporadically by the licensed (primary) users in legacy networks [1][2]. However, cognitive radio networks and their users, in general, may not have accurate and complete knowledge of spectrum availability. Therefore, their transmissions are inherently opportunistic, and as a key requirement, they should not introduce significant interference on the primary users' transmissions. This poses a fundamental challenge on how to schedule unlicensed (secondary) users' transmissions so as to ensure that interference constraints are respected and spectrum is optimally utilized. The challenge becomes more evident in scenarios where users dynamically join and leave the network, and in particular, where the total input load is not supportable.

In this work, we study how to reach the performance limit of such volatile networks, by introducing a novel admission control and scheduling policy, and demonstrating its optimality in terms of an arbitrary throughput utility function. We consider a widely deployed secondary (cognitive) network co-existing with a number of primary (licensed) networks. The secondary network consists of many Sub-Networks (SNs), where each $\mathrm{SN}$ is controlled by a Secondary Access Point (SAP) [3]. We assume that SAPs have only some side information about the availability of spectrum. The secondary users in each SN are further classified into Micro-Networks (MNs) based on their utility function, access capabilities, and/or arrival rate. The MNs under consideration can be maintained either for individual user data delivery or for collaborating users that communicate

\footnotetext{
The authors are with the Department of Electrical and Computer Engineering, University of Toronto. E-mail: \{mlotfinezhad, liang\}@comm.utoronto.ca, es.sousa@utoronto.ca. This work was supported in part by an NSERC Discovery Grant (RGPIN 262018).
}

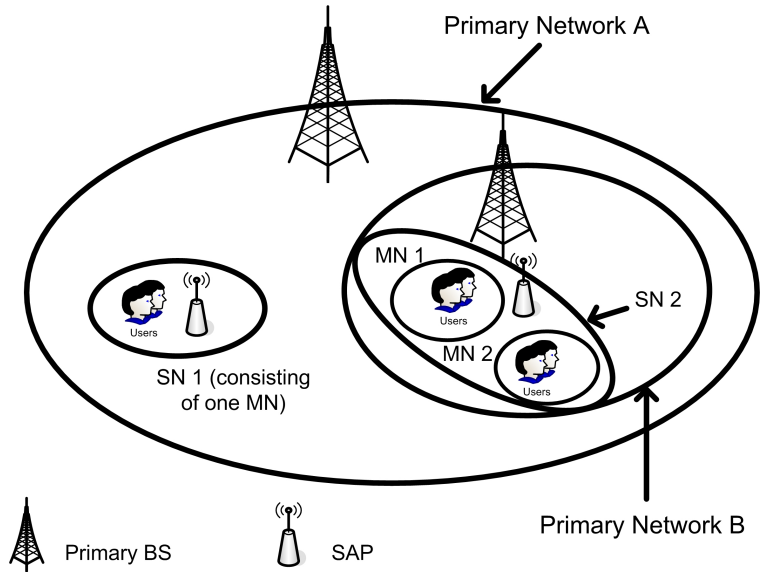

Fig. 1. Example of a coginitive radio network coexiting with primary networks $A$ and $B$.

to their SAP for a common purpose (e.g., as in a sensor network). In the latter case, we assume that there is an average power constraint. Furthermore, in contrast to previous works, we consider a network with dynamic population, where users bring a file (data) for transfer upon arrival to MNs, and once their file is transferred, they leave the network. See Fig. 1 for a simple example of the network model.

The proposed admission control and scheduling policy aims to maximize the aggregate throughput utility, subject to a set of constraints on the average interference received by primary networks and a set of average power constraints for MNs with collaborating users. This policy does not require the knowledge of input load and, by taking proper admission decisions, works for any load either inside or outside the capacity region [4]. We show that the policy yields aggregate throughput utility that is arbitrarily close to optimality, for any differentiable nondecreasing concave utility function. In particular, we prove that it is within $O(\delta)$ of the optimal performance for any positive $\delta$. We show that this arbitrarily closeness comes at the price of having a networking delay that is $O\left(\frac{1}{\delta}\right)$. As the policy can be in general complex, we further consider sub-optimal policies that are amenable to distributed implementation. We show that these policies can guarantee a $\frac{1}{\Delta}$-fraction of the optimal performance, where $\Delta$ is the maximum degree of the induced interference graphs.

The rest of this paper is organized as follows. In Section III, we describe the system model in detail. In Section IV, we formulate the throughput utility optimization problem. We present the admission control and scheduling policy and elaborate on its performance in Section V. A proof-sketch of the main theorem on policy performance is given in Section VI. In 
Section VII, we discuss approximations of the developed policy and characterize their performance. Finally, in Section VIII, we conclude the paper.

\section{RELATED WORK}

The literature is rich in various aspects of cognitive radio networking including the detection, access, and sharing of spectrum opportunities [1][2]. For instance, problems related to channel measurement and probing have been investigated in [5][6], and utility maximization has been the focus of many investigations, including game theory to design pricing schemes [7][8] and auction based spectrum sharing schemes [9].

In a more related context, [10] considers the problem of minimizing the required network-wide spectrum resource for a set of user sessions. The work [11] demonstrates that throughput maximization of a secondary user can be formulated as a constrained Partially Observable Markov Decision Process. Throughput maximization subject to long-term and maximum collision constraints are treated in [12] and in [13], respectively. In [3], the statistical distribution of spectrum demand is used to maximize the throughput utility of secondary users. Finally, in terms of sub-optimal implementation, distributed approaches to spectrum allocation for mobile ad-hoc networks are investigated in [14], and in [15], the authors provide a general approximation methodology through vertex labeling for channel assignment to maximize overall system utility. All these works either consider static networks or assume the availability of statistical knowledge of demand.

Our work takes inspiration from [13] and relies on a set of Lyapunov optimization tools similar to those used in [13]. However, our work is substantially different. First, while [13] focuses on throughput maximization with a fixed number of secondary users, our focus is on general throughput utility maximization in networks with dynamic user population. Second, we assume users may collaborate, under constraints on their power consumption. Third, we consider a generalized set of interference constraints. Finally, we show that, due to the different models considered, the scheduling problem in our setup is directly reducible to the Maximum Weighted Independent Set (MWIS) problem as opposed to the Maximum Weighted Matching (MWM) problem in [13 $]^{1}$.

\section{SYSTEM MODEL}

We consider a time-slotted system. Our goal is to model a generic network with both ordinary and collaborating users, while considering the interference constraints, transmission power constraints, user arrivals and departures, and the amount of available network state information. We show in the subsequent sections that throughput utility maximization for this network admits an elegant optimal admission control and scheduling solution.

We assume that the available frequency spectrum consists of $N_{b}$ orthogonal physical bands indexed by the set $\mathcal{N}_{b}=$ $\left\{1, \cdots, N_{b}\right\}$. These bands are used by a number of primary

\footnotetext{
${ }^{1}$ As a further indication of the modeling differences, the proposed policy achieves a performance that is within $O(\delta)$ of optimality as opposed to $O\left(\delta \log \left(\delta^{-1}\right)\right)$ in [13].
}

(legacy) wireless networks that provide service to primary users. We assume that each primary network, e.g., a single cell site covered by a base station, uses a subset of the available physical bands and covers a geographic area. We define each band used in a given primary network as one primary channel for this network. As a consequence of frequency reuse, two primary channels in different primary networks may be mapped into the same physical band. We define $N_{p}$ as the total number of primary channels summed over all primary networks and $\mathcal{N}_{p}=\left\{1, \ldots, N_{p}\right\}$ as the set indexing the primary channels.

To model a general network of secondary users, we assume that there are $M$ secondary SNs, each of which may represent a different geographic area with a certain user traffic load. We assume that each SN is controlled by a SAP. In general, SAPs assign spectrum to the users in their SN based on the availability of physical bands. We classify users in each SN according to the set of their accessible physical bands, their utility functions, and their mutual interference with primary and other secondary users. As a result, we apply all admission control and scheduling decisions to the classes to which users belong.

A class $j$ of users in $\mathrm{SN} i$ form an $\mathrm{MN}(i, j)$. For notational simplicity, we define a one-to-one function $l(i, j)$ that maps a given pair $(i, j)$ to a positive integer index, and hereafter, we use $l$ as the index for MNs. Let $\mathcal{L}=\{1, \ldots, L\}$ be the index set of all MNs. We assume that users belonging to $\mathrm{MN}$ $l$ are restricted to use the physical bands in the set $\mathcal{M}_{l}$, where $\mathcal{M}_{l} \subset \mathcal{N}_{b}$. Suppose users for any MN $l$ arrive according to an i.i.d process with mean rate $\lambda_{l}$, and each such user has a file for transfer with average size $\frac{1}{\mu_{l}}$. According to the preceding definitions, the average load associated with $\mathrm{MN} l$ is given by

$$
\rho_{l}=\frac{\lambda_{l}}{\mu_{l}} .
$$

Let $\boldsymbol{\rho}$ be the vector of input loads, i.e., $\boldsymbol{\rho}=\left(\rho_{1}, \ldots, \rho_{L}\right)$. We do not put any assumption on whether or not the load vector $\rho$ is inside the capacity region of the secondary network. Our proposal takes necessary actions to support the optimal part of $\rho$ (see (16)). We assume that file sizes for all MNs are restricted to belong to a finite set $\mathcal{F}=\left\{\frac{1}{f_{i}} ; 1 \leq i \leq F\right\}$, where $\frac{1}{f_{k}} \leq \frac{1}{f_{j}}$ if $k \leq j$. Furthermore, we assume that the file that a user brings for transfer in $\mathrm{MN} l$ has a random size with distribution $\left\{\pi_{l, k}\right\}$ , and we define $\rho_{l, k}$ as the load contributed by users with file size $\frac{1}{f_{k}}$ :

$$
\rho_{l, k}=\lambda_{l} \frac{\pi_{l, k}}{f_{k}} .
$$

To facilitate the optimization framework presented in the next section, we define the utility $U_{l}(\cdot)$ to be any differentiable nondecreasing concave function of the file size associated with $\mathrm{MN}$ $l$.

\section{A. Interference Model}

To model the interference between concurrent transmissions, we define the collision parameter $\gamma_{l_{1}, n}^{l_{2}} \in\{0,1\}$ to be one if and only if the transmission of (secondary) users in MN $l_{1}$ on band $n$ leads to packet losses of (secondary) users in MN $l_{2}$ on band $n$. Similarly, we define $\eta_{j}^{l, n} \in\{0,1\}$ to be 
one if and only transmissions by primary users on primary channel $j$ interfere with the transmission of users in $\mathrm{MN} l$ on band $n$. Finally, to model the interference effect of secondary transmissions on primary channels, we define $\eta_{l, n}^{j}$ to be the average path loss such that the interference power received on primary channel $j$ is $\eta_{l, n}^{j} P_{l, n}$ when a user in MN $l$ transmits with power $P_{l, n}$ on band $n$. This definition for $\eta_{l, n}^{j}$ is mainly motivated by the fact that primary receivers, e.g., base stations, are typically assumed to be sophisticated and can tolerate a certain level of interference power as long as it rarely goes beyond a given threshold [2].

\section{B. Primary Channel and Frequency Band States}

A SAP marks a physical band as available for one of its MNs if all primary channels interfering with the given physical band in the given $\mathrm{MN}$ are idle. Hence, the availability of a physical band depends on the state of interfering primary channels. Let $s_{j}^{(p)}(t) \in\{0,1\}$ be the state of primary channel $j$ at time $t$, where 1 denotes busy and 0 denotes idle. Let $\mathbf{s}^{(p)}(t)$ be the vector of all primary channel states, i.e.,

$$
\mathbf{s}^{(p)}(t)=\left(s_{1}^{(p)}(t), \ldots, s_{N_{p}}^{(p)}(t)\right),
$$

and let $\mathcal{S}^{(p)}$ be the set of all possible primary channel state vectors. Similarly, for a given set of primary channels $\Omega_{j} \subset \mathcal{N}_{p}$, we define $s_{\Omega_{j}}^{(p)}(t)$ to be one if all channels in $\Omega_{j}$ are busy and define $s_{\Omega_{j}}^{(p)}(t)$ to be zero, otherwise. Hence,

$$
s_{\Omega_{j}}^{(p)}(t)=\prod_{i \in \Omega_{j}} s_{i}^{(p)}(t) .
$$

We assume that $\mathbf{s}^{(p)}(t)$ is a convergent process in that for any given $\epsilon>0$, there exists $K_{\epsilon}^{\mathcal{S}^{(p)}}$ such that for $K \geq K_{\epsilon}^{\mathcal{S}^{(p)}}$, the following holds:

$$
\sum_{\mathbf{s}^{(p)} \in \mathcal{S}^{(p)}}\left|\mathbb{E}\left[\mathbf{1}_{\mathbf{s}^{(p)}(t+K)=\mathbf{s}^{(p)}} \mid \mathcal{H}(t)\right]-\pi_{\mathbf{s}^{(p)}}\right|<\epsilon,
$$

where $\mathbf{1}_{(\cdot)}$ is the indicator function, $\mathcal{H}(t)$ is the history of the primary channels process up and including time $t$, and $\pi_{\mathbf{s}(p)}$ is the steady-state probability of the channel at state $\mathbf{s}^{(p)}$.

Note that the availability spectrum is a local concept for each MN. In addition, what MNs care and can actually sense, perhaps with some error probability, is whether or not a physical band is idle. Therefore, we define $s_{l, n}(t) \in\{0,1\}$ as the state of physical band $n$ at time $t$ in $\mathrm{MN} l$. It is one if and only if physical band $n$ is busy from the viewpoint of MN $l$. Physical band $n$ is idle from the viewpoint of $\mathrm{MN} l$ if no primary channel that can interfere with $\mathrm{MN} l$ on band $n$ is transmitting at time $t$. Hence, we have

$$
s_{l, n}(t)=\left\{\begin{array}{ll}
0 & \text { if } \sum_{j=1}^{N_{p}} \eta_{j}^{l, n} s_{j}^{(p)}(t)=0 \\
1 & \text { otherwise }
\end{array} .\right.
$$

We define $\mathbf{s}_{l}(t)$ as the vector of band states from the view-point of $\mathrm{MN} l$ at time $t$, i.e., $\mathbf{s}_{l}(t)=\left(s_{l, 1}(t), \ldots, s_{l, N_{b}}(t)\right)$. We also define $\mathbf{s}^{(c)}(t), c$ for cognitive, to be the vector containing all $\mathbf{s}_{l}(t)$ 's:

$$
\mathbf{s}^{(c)}(t)=\left(\mathbf{s}_{1}(t), \ldots, \mathbf{s}_{L}(t)\right) .
$$

Let $\mathcal{S}^{(c)}$ be the set containing all possible values for $\mathbf{s}^{(c)}(t)$.
Based on these definitions, $\mathbf{s}^{(c)}(t)$ completely determines the state of all physical bands for all MNs.

Finally, we define $\mathbf{s}(t)$ to be the channel-band state as

$$
\mathbf{s}(t)=\left(\mathbf{s}^{(p)}(t), \mathbf{s}^{(c)}(t)\right)
$$

We define $\mathcal{S}$ to be the set containing all possible values for all $\mathbf{s}(t)$ 's.

\section{Channel-band State Side Information}

In general, SAPs may have access to only some side information about the current channel-band state of the network $\mathbf{s}(t)$. Denote the side information available to SAPs at time $t$ by $\psi(t)$, where $\psi(t)$ is in a finite set $\Psi$. For example, the side information $\psi(t)$ can be the delayed information, e.g., $\mathbf{s}^{(p)}(t-1)$, about the state of primary channels that is provided by the primary networks. In this case, SAPs can use the channel history to predict the probabilities defined below by (4) and (5). $\psi(t)$ may also contain the results of sensing and detection made by SAPs. Given $\psi(t)$, for a set of primary channels $\Omega_{j} \subset \mathcal{N}_{p}$, the probability that all channels in $\Omega_{j}$ are busy at time $t$ is given by

$$
p_{\Omega_{j}}^{(p)}(t) \triangleq \mathbb{E}\left[\mathbf{1}_{s_{\Omega_{j}}^{(p)}(t)=1} \mid \psi(t)\right]=\mathbb{E}\left[s_{\Omega_{j}}^{(p)}(t) \mid \psi(t)\right],
$$

where $s_{\Omega_{j}}^{(p)}(t)$ is defined in (1). Similarly, given $\psi(t)$, the probability that band $n$ is busy from the viewpoint of $\mathrm{MN} l$ is given by

$$
p_{l, n}(t) \triangleq \mathbb{E}\left[\mathbf{1}_{s_{l, n}(t)=1} \mid \psi(t)\right]=\mathbb{E}\left[s_{l, n}(t) \mid \psi(t)\right] .
$$

Note that the exact method for SAPs to obtain $\psi(t)$ is an open challenge [1][2] and is outside the scope of this paper. Considering that $\psi(t)$ is in general a (random) function of the process $\mathbf{s}(t)$, we assume that similar to the processes $\mathbf{s}^{(p)}(t)$, the composite process $(\psi(t), \mathbf{s}(t))$ is also convergent (see (2)). We also assert a sufficiency assumption that at any time $t$ all side information about $\mathbf{s}(t)$ is contained in $\psi(t)$. For example, this is the case when $\psi(t)=\mathbf{s}^{(p)}(t-1)$ and the primary channel state $\mathbf{s}^{(p)}(t)$ is a Markov chain. Finally, we assume there exits a $\kappa>0$ such that if $p_{\Omega_{j}}^{(p)}(t)>0$, then

$$
p_{\Omega_{j}}^{(p)}(t)>\kappa .
$$

It is easy to see that for the above Markovian example, a finite set $\Psi$ implies the existence of a positive $\kappa$.

\section{User Queueing: Schedules, Rates, and Powers}

Let $A_{l, k}(t)$ be the number of users arriving to $\mathrm{MN} l$ for timeslot $t$ with load $\frac{1}{f_{k}}$. We assume there exists a sufficiently large $A_{\max }$ such that for all $t$ and $l, \sum_{1 \leq k \leq F} A_{l, k}(t) \leq A_{\max }$. Then, it is clear that

$$
\sum_{k=1}^{F} \frac{A_{l, k}(t)}{f_{k}} \leq \tilde{A}_{\max } \triangleq \frac{A_{\max }}{f_{F}} .
$$

Let $r_{l, k}(t)$ be the number of such users with load $\frac{1}{f_{k}}$ that are admitted to $\mathrm{MN} l$ at time $t$, where $r_{l, k}(t) \leq A_{l, k}(t)$. Let $\varrho_{l, k}(t)=\frac{r_{l, k}(t)}{f_{k}}$ be the associated admitted load. The corresponding average number of admitted users and the average 
admitted load are given by

$$
\bar{r}_{l, k} \triangleq \lim _{T \rightarrow \infty} \frac{1}{T} \sum_{t=0}^{T-1} r_{l, k}(t) \text { and } \bar{\varrho}_{l, k} \triangleq \frac{\bar{r}_{l, k}}{f_{k}},
$$

respectively. We assume the decision on how many users to admit to each MN is taken by the MN's associated SAP. Using the definitions for $\varrho_{l, k}(t)$ and $\bar{\varrho}_{l, k}$, we define the total admitted load to $\mathrm{MN} l$ and its time-average as

$$
\varrho_{l}(t) \triangleq \sum_{k=1}^{F} \varrho_{l, k}(t) \text { and } \bar{\varrho}_{l} \triangleq \sum_{k=1}^{F} \bar{\varrho}_{l, k}=\sum_{k=1}^{F} \frac{\bar{r}_{l, k}}{f_{k}},
$$

respectively. In addition, we define the vector of the average admitted loads as

$$
\bar{\varrho} \triangleq\left(\bar{\varrho}_{1}, \ldots, \bar{\varrho}_{L}\right)
$$

To characterize scheduling decisions, let $I_{l, n}(t) \in\{0,1\}$ be one if and only $\mathrm{MN} l$ is assigned band $n$ by its associated SAP at time $t$. Hence, for a given time $t$, the vector

$$
\mathbf{I}(t) \triangleq\left(I_{1,1}(t), \ldots, I_{1, N_{b}}(t), \ldots, I_{L, 1}(t), \ldots, I_{L, N_{b}}(t)\right)
$$

specifies all scheduling actions at time $t$. Furthermore, we assume that $P_{l, n}$ units of power is used for transmission on band $n$ in $\mathrm{MN} l$, and if the transmission is successful, $R_{l, n}$ units of data can be transmitted. The transmission is successful if the following conditions are met. First, $s_{l, n}(t)=0$, which indicates that the transmission over band $n$ in $\mathrm{MN} l$ does not receive interference from primary networks. Second, $\mathrm{MN} l$ does not receive interference from any other $\mathrm{MN}$, which is equivalent to having $I_{l_{1}, n}(t) \gamma_{l_{1}, n}^{l}=0$, for all $l_{1} \neq l$.

Let $Q_{l}(t)$ be the backlog in terms of the total amount of data awaiting transmission in $\mathrm{MN} l$ at the beginning of time-slot $t$. Considering the above discussion, provided that

$$
\begin{aligned}
& r_{l, k}(t) \leq A_{l, k}(t), 1 \leq k \leq F \\
& I_{l, n}(t) I_{l_{1}, n}(t) \gamma_{l_{1}, n}^{l}=0, \quad \forall n \in \mathcal{N}_{b}, \forall l, l_{1}, l_{1} \neq l,
\end{aligned}
$$

for $l \in \mathcal{L}$, we have

$$
Q_{l}(t+1)=\left[Q_{l}(t)-D_{l}(t)\right]^{+}+\sum_{k=1}^{F} \frac{r_{l, k}(t)}{f_{k}},
$$

where $D_{l}(t)$ is the amount of transmitted data:

$$
D_{l}(t)=\sum_{n \in \mathcal{M}_{l}} R_{l, n} I_{l, n}(t)\left(1-s_{l, n}(t)\right) .
$$

Clearly, we have

$$
D_{l}(t) \leq R_{\max } \triangleq \max _{l \in \mathcal{L}} \sum_{n \in \mathcal{M}_{l}} R_{l, n}
$$

Without loss of generality, we assume that $Q_{l}(t)$ is a FIFO queue where admitted secondary users in $\mathrm{MN} l$ use the assigned bands on a first-come first-serve basis.

\section{Utility Optimization Problem}

To formulate the utility optimization problem for arbitrary utility functions, we consider two sets of MNs. Let $\mathcal{L}_{t} \subset \mathcal{L}$ be the first set consisting of typical MNs that are simply meant for user data delivery. Since for each of these MNs, utility is the user's level of satisfaction, the emphasis is on individual users.
Therefore, for $\mathrm{MN} l \in \mathcal{L}_{t}$, we define the long-term utility as

$$
\begin{aligned}
\bar{U}_{l} & =\lim _{T \rightarrow \infty} \frac{1}{T} \sum_{t=1}^{T} \sum_{k=1}^{F} r_{l, k}(t) U_{l}\left(\frac{1}{f_{k}}\right) \\
& =\sum_{k=1}^{F} \bar{r}_{l, k} U_{l}\left(\frac{1}{f_{k}}\right)=\sum_{k=1}^{F} \bar{\varrho}_{l, k} f_{k} U_{l}\left(\frac{1}{f_{k}}\right),
\end{aligned}
$$

which is simply the time-average of utilities perceived by the users in MN $l$. As for the second set of MNs, we assume users collaborate for a common purpose, as in a sensor network, to transmit data to their SAP. Let $\mathcal{L}_{c}$ be the set of MNs with collaborating users, where $\mathcal{L}_{t} \cup \mathcal{L}_{c}=\mathcal{L}$. For the $\mathrm{MN} l \in \mathcal{L}_{c}$, since the emphasis is on the performance of $\mathrm{MN}$ as a whole, utility should naturally be a function of the average aggregate communication rate; hence, we may define its long-term utility as

$$
\bar{U}_{l}=\lim _{T \rightarrow \infty} U_{l}\left(\frac{1}{T} \sum_{t=1}^{T} \sum_{k=1}^{F} \frac{r_{l, k}(t)}{f_{k}}\right)=U_{l}\left(\bar{\varrho}_{l}\right) .
$$

Note that we allow concurrent $\mathcal{L}_{t}$-type and $\mathcal{L}_{c}$-type MNs in the system under consideration.

It remains to specify the constraints that must be considered to ensure that the primary channels are not affected by secondary transmissions excessively. We consider in this paper a set of generalized interference constraints. First, note that the interference power on primary channel $j \in \mathcal{N}_{p}$ is defined by

$$
C_{j}(t) \triangleq s_{j}^{(p)}(t) \sum_{l \in \mathcal{L}} \sum_{n \in \mathcal{M}_{l}} I_{l, n}(t) P_{l, n} \eta_{l, n}^{j} .
$$

where $s_{j}^{(p)}(t)$ appears in $C_{j}(t)$ since the interference affects the performance of channel $j$ only when the channel is active. We can further generalize this to the case where a primary session requires multiple channels simultaneously. Given a set of primary channels $\Omega_{j} \subset \mathcal{N}_{p}$, we define $C_{\Omega_{j}, i}(t)$ as

$$
C_{\Omega_{j}, i}(t)=s_{\Omega_{j}}^{(p)}(t) \sum_{l \in \mathcal{L}} \sum_{n \in \mathcal{M}_{l}} I_{l, n}(t) P_{l, n} \eta_{l, n}^{i} .
$$

Note that $s_{\Omega_{j}}^{(p)}(t)=1$, as defined in (1), can be a sign of instantaneous heavy and urgent traffic on all channels in $\Omega_{j}$. Therefore, by considering the generalized interference constraints in the form of (12), we can be more specific and better protect a variety of primary transmissions. Clearly, we have

$$
C_{\Omega_{j}, i}(t) \leq C_{\max } \triangleq \max _{\left\{i \mid \exists j, i \in \Omega_{j}\right\}} \sum_{l \in \mathcal{L}} \sum_{n \in \mathcal{M}_{l}} P_{l, n} \eta_{l, n}^{i} .
$$

Note that (13) includes the case for ordinary interference power on a single primary channel when $\Omega_{j}=\{j\}$.

To define the interference constraints, let $T_{\Omega_{j}}=$ $\sum_{t=0}^{T-1} s_{\Omega_{j}}^{(p)}(t)$; hence, $T_{\Omega_{j}}$ is the total number of timeslots up to and including timeslot $T-1$, within which all primary channels in $\Omega_{j}$ are busy at the same time. We assume the following constraint for each set $\Omega_{j}$ :

$$
\bar{C}_{\Omega_{j}, i} \triangleq \lim _{T \rightarrow \infty} \frac{1}{T_{\Omega_{j}}} \sum_{t=0}^{T-1} C_{\Omega_{j}, i}(t) \leq \hat{C}_{\Omega_{j}, i} .
$$

so that the average total interference is upper bounded by $\hat{C}_{\Omega_{j}, i}$. 
Note that dividing by $T_{\Omega_{j}}$ instead of $T$ ensures the same relative level of protection regardless of the primary load on primary channels in $\Omega_{j}$ [1][11]. We define $\hat{C}_{\max } \triangleq \max _{j, i \in \Omega_{j}} \hat{C}_{\Omega_{j}, i}$.

Average interference constraints are widely used in the literature [1][12][13]. Nevertheless, our analysis framework can be easily modified to allow replacement of the constraint in (14) with one that limits the fraction of time that the interference $C_{\Omega_{j}, i}(t)$ exceeds a given threshold ${ }^{2}$. In this way, when the fraction of time is made very small, the constraint can be reduced to a special case approximating the instantaneous maximum interference bound.

As discussed earlier, an $\mathrm{MN}$ might represent a network with collaborating users as in a sensor network. In such a case, it is important to avoid excessive transmissions to maintain the average power consumption below a given limit. In general, we may assume a power constraint for a given set of MNs, which should naturally belong to the same $\mathrm{SN}$. In particular, given a set of MNs $\Phi_{m} \subset \mathcal{L}$, we define $P_{\Phi_{m}}(t)$ as the total power used in $\Phi_{m}$ at time $t$ :

$$
\begin{aligned}
P_{\Phi_{m}}(t) & =\sum_{l \in \Phi_{m}, n \in \mathcal{M}_{l}} I_{l, n}(t) P_{l, n} \\
& \leq P_{\max } \triangleq \max _{m} \sum_{l \in \Phi_{m}, n \in \mathcal{M}_{l}} P_{l, n} .
\end{aligned}
$$

We consider an upper bound $\hat{P}_{\Phi_{m}}$ for the time average of the power consumption over all MNs in $\Phi_{m}$ :

$$
\bar{P}_{\Phi_{m}} \triangleq \lim _{T \rightarrow \infty} \frac{1}{T} \sum_{t=0}^{T-1} P_{\Phi_{m}}(t) \leq \hat{P}_{\Phi_{m}} .
$$

We define $\hat{P}_{\text {max }}=\max _{m} \hat{P}_{\Phi_{m}}$ and $\Xi_{l}=\left\{m \mid l \in \Phi_{m}\right\}$. Therefore, $\Xi_{l}$ represents all $\Phi_{m}$ 's that contain MN $l$.

Considering the objective functions in (10) and (11), and constraints introduced earlier in (14) and (15), in this paper, we are interested in the following optimization problem:

$$
\begin{array}{ll}
\text { Maximize } & \sum_{l \in \mathcal{L}_{t}} \sum_{k=1}^{F} \bar{\varrho}_{l, k} f_{k} U_{l}\left(\frac{1}{f_{k}}\right)+\sum_{l \in \mathcal{L}_{c}} U_{l}\left(\bar{\varrho}_{l}\right) \\
\text { Subject to: } \quad & \bar{C}_{\Omega_{j}, i} \leq \hat{C}_{\Omega_{j}, i}, 1 \leq j \leq N_{\Omega}, \quad i \in \Omega_{j}, \\
& \bar{P}_{\Phi_{m}} \leq \hat{P}_{\Phi_{m}}, 1 \leq m \leq N_{\Phi}, \\
& \bar{\varrho}_{l} \leq \rho_{l}, 1 \leq l \leq L, \\
& \bar{\varrho} \in \Gamma,
\end{array}
$$

where $N_{\Omega}$ and $N_{\Phi}$ are respectively the numbers of $\Omega_{j}$ 's and $\Phi_{m}$ 's under consideration, and $\Gamma$ is the network-layer capacity region [4]. Note that even though $N_{\Omega}$ and $N_{\Phi}$ potentially can be up to $2^{N_{p}}$ and $2^{L}$ respectively, in reality they are are far smaller. For example, in the common scenario where the sets $\Omega_{j}$ partition $\mathcal{N}_{p}$, there are at most $N_{p}$ such sets. We label the optimal solution to the above problem by $\bar{\varrho}_{l, k}^{*}$ and $\bar{\varrho}_{l}^{*}$.

\footnotetext{
${ }^{2}$ We need to simply keep track of the fraction of time, e.g., by counting via a simple indicator function, that interference has gone beyond the threshold, based on which we make scheduling decisions. In this case, we need an additional virtual queue for this fraction (see Section V.A), similar to those used to make sure the average interference and power are kept below a threshold.
}

\section{AdMission CONTROL AND SCHEdULING}

We elaborate on the structure of the proposed Admission Control and Scheduling (ACS) policy, denoted by $\Upsilon^{A C S}$ to solve (16). As desired, this policy does not require the knowledge of input load vector $\rho$, neither its statistics nor whether or not it is inside the region $\Gamma$.

\section{A. Preliminary: Control Queues}

The policy $\Upsilon^{A C S}$ makes novel uses of three sets of control queues ${ }^{3}$. The first set contains a reverse queue $X_{l}(t)$, for each $l \in \mathcal{L}_{c}$, where $X_{l}(t)$ is updated, by the SAP associated with MN $l$, as follows:

$$
X_{l}(t+1)=\left[X_{l}(t)-\sum_{k=1}^{F} \frac{r_{l, k}(t)}{f_{k}}\right]^{+}+\xi_{l}(t),
$$

where $\xi_{l}(t) \leq \xi_{\max } \triangleq 2 \tilde{A}_{\max }$ is the reverse control variable for MN l, which, as discussed in Section V-B.1, is updated by $\Upsilon^{A C S}$. Note that $X_{l}(t)$ is a reverse queue since arrivals to $Q_{l}(t)$ are departures from $X_{l}(t)$.

The second and third sets of control queues are the interference queues $\left\{Y_{j, i}(t), 1 \leq j \leq N_{\Omega}, i \in \Omega_{j}\right\}$ and power queues $\left\{Z_{m}(t), 1 \leq m \leq N_{\Phi}\right\}$, where $Y_{j, i}(t)$ and $Z_{m}(t)$ are updated as

$$
\begin{aligned}
& Y_{j, i}(t+1)=\left[Y_{j, i}(t)-s_{\Omega_{j}}^{(p)}(t) \hat{C}_{\Omega_{j}, i}\right]^{+}+C_{\Omega_{j}, i}(t), \\
& Z_{m}(t+1)=\left[Z_{m}(t)-\hat{P}_{\Phi_{m}}\right]^{+}+\sum_{l \in \Phi_{m}, n \in \mathcal{M}_{l}} I_{l, n}(t) P_{l, n} .
\end{aligned}
$$

It is easy to see that if these queues are stable, all interference and power constraints are satisfied. Note that both $X_{l}(t)$ and $Z_{m}(t)$ can be maintained at the SAP responsible for MN $l$ and the set $\Phi_{m}$, respectively. The value of interference queue $Y_{j, i}(t)$, on the other hand, is based on the received interference, which we assume is provided by the primary network to SAPs.

\section{B. Policy $\Upsilon^{A C S}$}

The policy $\Upsilon^{A C S}$ uses a control parameter $\delta>0$ that can be arbitrarily selected to tradeoff delay for utility performance. This policy consists of two parts as follows (see Fig. 2 for an overview of network operation under $\Upsilon^{A C S}$ ).

1) Admission Control: At each timeslot $t$, if $l \in \mathcal{L}_{t}$, choose $r_{l, k}(t), 1 \leq k \leq F$, as the number of admitted users with file size $\frac{1}{f_{k}}$ according to the following optimization problem:

$$
\begin{aligned}
& \text { Minimize: }\left[\left(\frac{Q_{l}(t)}{f_{k}}-\delta^{-1} U_{l}\left(\frac{1}{f_{k}}\right)\right) r_{l, k}(t)\right], \\
& \text { Subject to: } 0 \leq r_{l, k}(t) \leq A_{l, k}(t) .
\end{aligned}
$$

If $l \in \mathcal{L}_{c}$, choose $r_{l, k}(t)$ 's collectively as the solution to the following problem:

$$
\begin{aligned}
& \text { Minimize: }\left[\left(Q_{l}(t)-X_{l}(t)\right) \sum_{k=1}^{F} \frac{r_{l, k}(t)}{f_{k}}\right], \\
& \text { Subject to: } 0 \leq r_{l, k}(t) \leq A_{l, k}(t), \quad 1 \leq k \leq F
\end{aligned}
$$

\footnotetext{
${ }^{3}$ These queues are virtual since they are implemented solely for the network control purposes [16].
} 


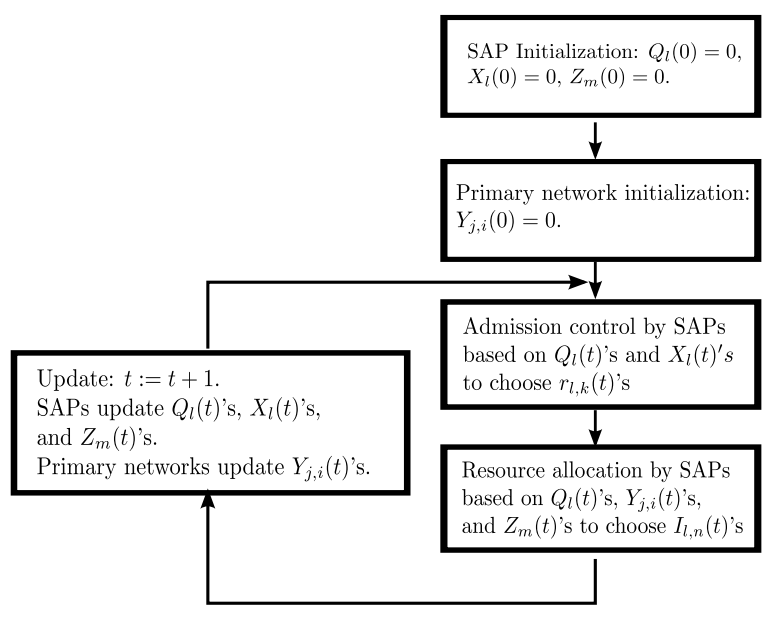

Fig. 2. Logical chart of the network operation under ACS policy.

In addition, for $l \in \mathcal{L}_{c}$, the reverse control variables $\xi_{l}(t)$ 's are selected to solve

$$
\begin{aligned}
& \text { Minimize: }\left[X_{l}(t) \xi_{l}(t)-\delta^{-1} U_{l}\left(\xi_{l}(t)\right)\right], \\
& \text { Subject to: } \xi_{l}(t) \leq \xi_{\max } .
\end{aligned}
$$

2) Resource Scheduling: At each timeslot $t$, choose the schedule vector $\mathbf{I}(t)$ that is the solution to the following optimization problem:

$$
\text { Maximize: }\left[\sum_{l \in \mathcal{L}} \sum_{n \in \mathcal{M}_{l}} I_{l, n}(t) w_{l, n}(t)\right],
$$

Subject to: the constraint in (8),

where the wieght $w_{l, n}(t)$ is given by

$$
\begin{aligned}
& w_{l, n}(t)=Q_{l}(t) R_{l, n}\left(1-p_{l, n}(t)\right) \\
& \quad-P_{l, n}\left(\sum_{1 \leq j \leq N_{p}} \sum_{i \in \Omega_{j}} p_{\Omega_{j}}^{(p)}(t) Y_{j, i}(t) \eta_{l, n}^{i}+\sum_{m \in \Xi_{l}} Z_{m}(t)\right),
\end{aligned}
$$

and $p_{\Omega_{j}}^{(p)}(t)$ and $p_{l, n}(t)$ are given in (4) and (5), respectively, and $\Xi_{l}$ is defined after (15).

\section{Intuitive Explanation of the Policy}

The admission control for each MN solves one or two simple optimization problems. Specifically, when $l \in \mathcal{L}_{t}$, it reduces to a simple threshold rule, where all $A_{l, k}(t)$ users with file size $\frac{1}{f_{k}}$ are admitted if $Q_{l}(t) \leq \delta^{-1} f_{k} U_{l}\left(\frac{1}{f_{k}}\right)$, otherwise all such users are dropped and $r_{l, k}(t)$ is set to zero. Similarly, for $l \in \mathcal{L}_{c}$, all arriving users are admitted if $Q_{l}(t) \leq X_{l}(t)$, otherwise for all $k, r_{l, k}(t)$ is set to zero. Additionally, for $l \in \mathcal{L}_{c}$, the reverse control variable is chosen to minimize a simple convex function. For instance, if $U_{l}(\xi)=\log (1+\xi)$, we have

$$
\xi_{l}(t)=\min \left[\max \left[0, \frac{1}{\delta X_{l}(t)}-1\right], \xi_{\max }\right] .
$$

Note that all minimizations in the admission control for MN $l$ require only local data of that MN available at its SAP. Hence, admission control, which is implemented in the SAP corresponding to each $\mathrm{MN}$, is decentralized over SAPs.

The main difficulty in implementing policy $\Upsilon^{A C S}$ lies in solving the resource allocation problem of (20). To convert this problem to a known optimization problem, for simplicity of illustration, suppose that interference is symmetric, such that $\gamma_{l, n}^{l_{1}}=\gamma_{l_{1}, n}^{l}$, for all $l$ and $l_{1}$ in $\mathcal{L}$. Let each band $n$ represent a color. Now, consider the colored graph $G(V, E, W)$, where $V=\mathcal{L}$ is the set of vertices representing MNs, $E$ is the set of edges representing interference between any two MNs, and $W$ is the set of weights $\left(w_{l, n}(t)^{\prime}\right.$ 's). Suppose there is an $n$-colored edge between $l_{1}$ and $l_{2}$ if $\gamma_{l_{1}, n}^{l_{2}}=1$. We assume that if a vertex $l$ is colored by color $n$, which happens when $I_{l, n}(t)=1$, it adds a weight of $w_{l, n}(t)$. The resource allocation problem then reduces to coloring each vertex ( $\mathrm{MN} l$ ) using a number of colors (bands) from its color list $\left(\mathcal{M}_{l}\right)$ such that the summation of gained weights is maximized and no two vertices connected by an $n$-colored edge use color $n$ simultaneously. In general where the number of selected colors (bands) is limited by a constant $M_{\max }$, this problem is recognized as Color-Sensitive Graph Coloring (CSGC) [15]. When vertices are free to choose any number of colors from their list, as in our case, it is easy to see that choosing $I_{l, n}(t)$ 's for a given $n \in \mathcal{N}_{b}$ is independent of choosing $I_{l, n_{1}}(t)$ 's for $n_{1} \neq n$. Hence, the problem in (20) decomposes into $N_{b}$ separate maximization problems each for a given band $n$. These problems are mono-color versions of CSGC, which are otherwise known as the Maximum Weighted Independent Set (MWIS) problem [15][17].

Both CSGC and MWIS are NP-hard in general [15]. Fortunately, there are simple greedy algorithms that for a given graph provide constant factor approximations for MWIS. In Section VII, we consider a general model for distributed algorithms, including the available greedy algorithms, that approximate the solution of (20), and study their performance.

\section{Main Theorem on Performance Characterization}

To state the theorem, let $\zeta_{1}=\max _{l \in \mathcal{L}_{c}} U_{l}^{\prime}(0)$ be the maximum derivative at zero, $\zeta_{2}=\max _{l \in \mathcal{L}_{t}, 1 \leq k \leq F} f_{k} U_{l}\left(\frac{1}{f_{k}}\right)$, and

$$
\begin{aligned}
B= & \frac{1}{2}\left(L\left(\tilde{A}_{\text {max }}^{2}+R_{\text {max }}^{2}\right)+N_{p}\left(C_{\text {max }}^{2}+\hat{C}_{\text {max }}^{2}\right)\right. \\
& \left.+L\left(P_{\text {max }}^{2}+\hat{P}_{\text {max }}^{2}\right)+3\left|\mathcal{L}_{c}\right| \tilde{A}_{\text {max }}^{2}\right) .
\end{aligned}
$$

In addition, let $g^{*}$ be the optimal value for the objective function of (16), i.e.,

$$
g^{*}=\sum_{l \in \mathcal{L}_{t}} \sum_{k=1}^{F} \bar{\varrho}_{l, k}^{*} f_{k} U_{l}\left(\frac{1}{f_{k}}\right)-\sum_{l \in \mathcal{L}_{c}} U_{l}\left(\bar{\varrho}_{l}^{*}\right) .
$$

We have the following theorem, whose proof is provided in Section VI.

Theorem 1. For any input load vector $\rho$, the policy $\Upsilon^{A C S}$ conforms to the interference and power constraints given in (16) and ensures the following performance bound:

$$
\begin{aligned}
\liminf _{T \rightarrow \infty}\left\{\sum_{l \in \mathcal{L}_{t}} \sum_{k=1}^{F}\left(\frac{1}{T} \sum_{t=0}^{T-1} \mathbb{E}\left[\varrho_{l, k}(t)\right]\right) f_{k} U_{l}\left(\frac{1}{f_{k}}\right)\right. \\
\left.\left.\quad+\sum_{l \in \mathcal{L}_{c}} \mathbb{E}\left[U_{l}\left(\frac{1}{T} \sum_{t=0}^{T-1} \varrho_{l}(t)\right]\right)\right]\right\} \geq g^{*}-\delta B,
\end{aligned}
$$

In addition, $\Upsilon^{A C S}$ stabilizes all queues including all control 
queues yielding the following deterministic bounds for $t \geq 0$ :

$$
\begin{aligned}
& X_{l}(t) \leq X_{\text {max }} \triangleq \delta^{-1} \zeta_{1}+\xi_{\text {max }} \\
& Q_{l}(t) \leq Q_{\text {max }} \triangleq \max \left(X_{\max }, \delta^{-1} \zeta_{2}\right)+\tilde{A}_{\text {max }}, \\
& Y_{j, i}(t) \leq Y_{\max } \triangleq \frac{Q_{\max }}{\kappa}\left(\max _{l \in \mathcal{L}, n \in \mathcal{M}_{l}} \frac{R_{l, n}}{P_{l, n}}\right)+C_{\max } \\
& Z_{m}(t) \leq Z_{\text {max }} \triangleq Q_{\max }\left(\max _{l \in \mathcal{L}, n \in \mathcal{M}_{l}} \frac{R_{l, n}}{P_{l, n}}\right)+P_{\text {max }} .
\end{aligned}
$$

This theorem shows that $\Upsilon^{A C S}$ provides an average utility performance that is within $O(\delta)$ of the optimal performance, for any $\delta>0$. The second part of the theorem, however, shows that the bound for $Q_{l}(t)$ 's increases with $\frac{1}{\delta}$ as $\delta \rightarrow 0$, suggesting a tradeoff between utility and the delay in admitting users. Hence, we have the following:

Corollary 1. Under $\Upsilon^{A C S}$, the avergae utility performance is within $O(\delta)$ of the optimality, and $Q_{l}(t)$ 's are $O\left(\delta^{-1}\right)$, where $\delta>0$ can be any positive real number.

It is worthy to note that when the coverage area of the cognitive radio network increases, the nubmer of SNs and, consequently, the number of MNs, $L$, increase. The parameter $B$ in the theorem, however, increases linearly with $L$. Hence, to make sure that the distance $\delta B$ to the optimal utility is fixed, $\delta$ should be proportional to $L^{-1}$, and therefore, based on the above, delay increases linearly with $L$ and the network coverage. However, it is likely that $g^{*}$ also increases linearly with $L$ as the coverage increases. If that is the case, then for a fixed $\delta$ and, as a result, an $O(1)$ delay, a fixed fraction $1-\frac{\delta B}{g^{*}}$ of the optimal utility $g^{*}$ can be achived.

In addition, note the queue bounds are deterministic and ensure that excess received interference and transmit power both are properly limited. Specifically, noting that $Y_{\max }$ and $Z_{\text {max }}$ both are $O\left(\delta^{-1}\right)$, and recalling that $\hat{C}_{\Omega_{j}, i}$ and $\hat{P}_{\Phi_{m}}$ are the given upper-bounds for the average inteference and power consumption, we have the following:

Corollary 2. Over any interval containing $K$ timeslots within which all physical channels in $\Omega_{j}$ are busy, the interference power on channel $i \in \Omega_{j}$ does not go beyond $K \hat{C}_{\Omega_{j}, i}+O\left(\delta^{-1}\right)$. Similarly, over any interval of size $K$, the total power consumed by MNs in $\Phi_{m}$ does not go beyond $K \hat{P}_{\Phi_{m}}+O\left(\delta^{-1}\right)$.

\section{Proof of Main Theorem}

We provide here the proof-sketch for the utility performancebound in Theorem 1. Further details and the proof for queue bounds and the subsequent propositions are provided in [18].

While adopting the general framework of Lyapunov Optimization developed in [19][4], the proof for the performance bound uses a novel result on Lyapunov optimization with variable drift. To establish the result, consider a queueing system with the vector of queues $\Theta(t)=\left(\theta_{1}(t), \ldots, \theta_{n}(t)\right)$. Let $L(t)=L(\Theta(t))$ be a non-negative (scalar) function of $\Theta(t)$ and define the drift function at time $t$ to be

$$
\Delta(L(t))=\mathbb{E}[L(t+1)-L(t)] .
$$

Suppose $g(t)$ is a well-defined utility function, perhaps as a function of actions taken at time $t$, and let $g^{*}$ be the target value for $g(t)$. We have the following:
Proposition 1. Suppose for $t \geq 0$, the following Lyapunov drift holds:

$$
\Delta(L(t))-\frac{1}{\delta} \mathbb{E}[g(t)] \leq B+B_{1} \epsilon_{t}-\frac{1}{\delta} g^{*},
$$

where $0 \leq B, B_{1}<\infty, \delta>0$, and the sequence $\left\{\epsilon_{t}\right\}$ is a (non-random) sequence of non-negative real numbers with the property that $\lim _{T \rightarrow \infty} \frac{1}{T} \sum_{t=0}^{T-1} \epsilon_{t}=0$. Then, we have that

$$
\liminf _{T \rightarrow \infty} \frac{1}{T} \sum_{t=0}^{T-1} \mathbb{E}[g(t)] \geq g^{*}-\delta B .
$$

The other key result required for the proof is the following:

Proposition 2. For any input load $\rho$, there exists a stationary scheduling policy $\Upsilon_{s}^{S T A T}$ that selects the schedule vector $\mathbf{I}(t)$ only as a function of $\psi(t)$ such that at the steady state the expected transmission rate $D_{l}^{S T A T}$ for $M N l, 1 \leq l \leq L$, the expected interference power $C_{\Omega_{j}, i}^{S T A T}, 1 \leq j \leq N_{\Omega}, i \in \Omega_{j}$, and the expected transmission power $P_{\Phi_{m}}^{S T A T}, 1 \leq m \leq N_{\Phi}$, satisfy:

$$
D_{l}^{S T A T} \geq \bar{\varrho}_{l}^{*}, C_{\Omega_{j}, i}^{S T A T} \leq \pi_{\mathbf{s}_{\Omega_{j}}^{(p)}=1} \hat{C}_{\Omega_{j}, i}, P_{\Phi_{m}}^{S T A T} \leq \hat{P}_{\Phi_{m}} .
$$

Proof-sketch of the performance bound: Consider the following Lyapunov function:

$$
L(t)=\frac{1}{2}\left(\sum_{l=1}^{L} Q_{l}^{2}(t)+\sum_{j=1}^{N_{\Omega}} Y_{j}^{2}(t)+\sum_{m=1}^{N_{\Phi}} Z_{m}^{2}(t)+\sum_{l \in \mathcal{L}_{c}} X_{l}^{2}(t)\right) .
$$

Our goal is to find an inequality suitable to apply Proposition 1. Using (9), (17), (18), and (19), we can show that

$$
\begin{aligned}
& \Delta(L(t)) \leq B+\mathbb{E}\left[\sum _ { l = 1 } ^ { L } Q _ { l } ( t ) \left(\sum_{k=1} \frac{r_{l, k}(t)}{f_{k}}-\sum_{n \in \mathcal{M}_{l}} R_{l, n} I_{l, n}(t)\right.\right. \\
& \left.\left(1-s_{l, n}(t)\right)\right)+\sum_{j=1}^{N_{\Omega}} \sum_{i \in \Omega_{j}} Y_{j, i}(t)\left(C_{\Omega_{j}, i}(t)-s_{\Omega_{j}}^{(p)}(t) \hat{C}_{\Omega_{j}, i}\right) \\
& +\sum_{m=1}^{N_{\Phi}} Z_{m}(t)\left(\sum_{l \in \Phi_{m}, n \in \mathcal{M}_{l}} I_{l, n}(t) P_{l, n}-P_{\hat{\Phi}_{m}}\right) \\
& \left.+\sum_{l \in \mathcal{L}_{c}} X_{l}(t)\left(\xi_{l}(t)-\sum_{k=1}^{F} \frac{r_{l, k}(t)}{f_{k}}\right)\right],
\end{aligned}
$$

where $B$ is defined in (21). Subtracting a $\delta^{-1}$-scaled version of

$$
g(t) \triangleq \mathbb{E}\left[\sum_{l \in \mathcal{L}_{t}} \sum_{k=1}^{F} r_{l, k}(t) U_{l}\left(\frac{1}{f_{k}}\right)+\sum_{l \in \mathcal{L}_{c}} U_{l}\left(\xi_{l}(t)\right)\right]
$$

from both sides of (25) and rearranging terms lead to

$$
\begin{aligned}
& \Delta(L(t))-\delta^{-1} \mathbb{E}[g(t)] \leq B+\mathbb{E}\left[\sum _ { l \in \mathcal { L } _ { t } } \sum _ { k = 1 } ^ { F } r _ { l , k } ( t ) \left(\frac{Q_{l}(t)}{f_{k}}\right.\right. \\
& \left.\left.-\delta^{-1} U_{l}\left(\frac{1}{f_{k}}\right)\right)\right]+\mathbb{E}\left[\sum_{l \in \mathcal{L}_{c}}\left(\left(Q_{l}(t)-X_{l}(t)\right) \sum_{k=1}^{F} \frac{r_{l, k}(t)}{f_{k}}\right)\right] \\
& \quad+\mathbb{E}\left[\sum_{l \in \mathcal{L}_{c}}\left(X_{l}(t) \xi_{l}(t)-\delta^{-1} U_{l}\left(\xi_{l}(t)\right)\right)\right]
\end{aligned}
$$




$$
\begin{aligned}
& -\mathbb{E}\left[\sum _ { l \in \mathcal { L } } \sum _ { n \in \mathcal { M } _ { l } } I _ { l , n } ( t ) \left(Q_{l}(t) R_{l, n}\left(1-s_{l, n}(t)\right)\right.\right. \\
& \left.\left.-P_{l, n}\left(\sum_{j=1}^{N_{\Omega}} \sum_{i \in \Omega_{j}} s_{\Omega_{j}}^{(p)}(t) Y_{j, i}(t) \eta_{l, n}^{i}+\sum_{m \in \Xi_{l}} Z_{m}(t)\right)\right)\right]- \\
& \mathbb{E}\left[\sum_{j=1}^{N_{\Omega}} \sum_{i \in \Omega_{j}} Y_{j, i}(t) s_{\Omega_{j}}^{(p)}(t) \hat{C}_{\Omega_{j}, i}+\sum_{m=1}^{N_{\Phi}} Z_{m}(t) \hat{P}_{\Phi_{m}}\right] .
\end{aligned}
$$

Examining (26) shows that the policy $\Upsilon^{A C S}$ minimizes the right hand side of (26) over all admissible policies that have access to and use only the side information $\psi(t)$ and queue information (control or not) for admission control and scheduling. To see this, note that for any given queue information, the arguments inside the first, second, and third expectations in the RHS of (26) are minimized by $\Upsilon^{A C S}$, as explained in Section V-B.1, so that their expected values are also minimized by $\Upsilon^{A C S}$. Similarly, by the definition of resource scheduling given in Section V-B.2 and the definition of $p_{\Omega_{j}}^{(p)}(t)$ and $p_{l, n}(t)$ given in (4) and (5), we see that $\Upsilon^{A C S}$ maximizes the expected value of the argument inside the forth expectation in the RHS of (26) for any given $\psi(t)$ and queue information at time $t$. Hence, using conditional expectations and taking into account the negative sign, we see that the forth expectation is also minimized by $\Upsilon^{A C S}$. Therefore, as stated, the policy $\Upsilon^{A C S}$ minimizes the RHS of (26) over all admissible policies.

The above observation provides a means to derive an upperbound for the LHS of (26). In particular, we first construct an admissible stationary policy $\Upsilon^{S T A T}$. We then evaluate the RHS of (26) when $\Upsilon^{S T A T}$ is used. By the above discussion, the evaluated RHS is larger than the one corresponding to $\Upsilon^{A C S}$, which in turn is larger than the LHS of (26). We construct $\Upsilon^{S T A T}$ as follows. We assume that $\Upsilon^{S T A T}$ uses the stationary scheduling policy $\Upsilon_{s}^{S T A T}$ specified in Proposition 2. For admission control, we assume $\Upsilon^{S T A T}$ performs the following. At any time $t$ and for $l \in \mathcal{L}_{t}$, with probability $\frac{\bar{\varrho}_{l, k}^{*}}{\rho_{l, k}}$, policy $\Upsilon^{S T A T}$ independently admits all arriving users with file size $\frac{1}{f_{k}}$ and sets $r_{l, k}(t)=A_{l, k}(t)$; otherwise, it sets $r_{l, k}(t)=0$. In a similar manner, for $l \in \mathcal{L}_{c}$, with probability $\frac{\bar{Q}_{l}^{*}}{\rho_{l}}$, policy $\Upsilon^{S T A T}$ sets $r_{l, k}(t)=A_{l, k}(t)$ for all $k$, i.e., admits all new users. Otherwise, the policy sets $r_{l, k}(t)=0$. Using (7), it is easy to show that for both $l \in \mathcal{L}_{t}$ and $l \in \mathcal{L}_{c}{ }^{4}$

$$
\mathbb{E}\left[\sum_{k=1}^{F} \frac{r_{l, k}(t)}{f_{k}} \mid \psi(t), \mathbf{Q}(t), \mathbf{Y}(t), \mathbf{Z}(t), \mathbf{X}(t)\right]=\bar{\varrho}_{l}^{*} .
$$

As for $\xi_{l}(t)$ 's, policy $\Upsilon^{S T A T}$ chooses a fixed value for each $\xi_{l}(t)$ and sets

$$
\xi_{l}(t)=\bar{\varrho}_{l}^{*}, l \in \mathcal{L}_{c} .
$$

Now that we have constructed $\Upsilon^{S T A T}$, we can use the discussion in the previous paragraph to find an upper bound for the LHS of (26). In particular, noting that the RHS of (26) is equal to the RHS of (25) minus $\delta^{-1} g(t)$, by (27)-(28), we

\footnotetext{
${ }^{4}$ Note that boldface is reserved for vectors. Hence, $\mathbf{Q}(t), \mathbf{Y}(t), \mathbf{Z}(t)$, and $\mathbf{X}(t)$ represent the vector of all $Q_{l}(t)$ 's, $Y_{j, i}(t)$ 's, $Z_{m}(t)$ 's, and $X_{l}(t)$ 's, respectively.
}

can evaluate the RHS of (26) for $\Upsilon^{S T A T}$ and show that

$$
\begin{aligned}
& \Delta(L(t))-\delta^{-1} \mathbb{E}[g(t)] \leq B+\mathbb{E}\left[\sum _ { l = 1 } ^ { L } Q _ { l } ( t ) \left(\bar{\varrho}_{l}^{*}-\right.\right. \\
& \underbrace{\sum_{n \in \mathcal{M}_{l}} R_{l, n} I_{l, n}^{S T A T}(t)\left(1-s_{l, n}(t)\right)}_{D_{l}^{S T A T}(t)})+\sum_{j=1}^{N_{\Omega}} \sum_{i \in \Omega_{j}} Y_{j, i}(t) \\
& \left(C_{\Omega_{j}, i}^{S T A T}(t)-s_{\Omega_{j}}^{(p)}(t) \hat{C}_{\Omega_{j}, i}\right)+\sum_{m=1}^{N_{\Phi}} Z_{m}(t) \\
& \left.(\underbrace{\sum_{l \in \Phi_{m}, n \in \mathcal{M}_{l}} I_{l, n}^{S T A T}(t) P_{l, n}}_{P_{\Phi_{m}}^{S T A T}(t)}-\hat{P}_{\Phi_{m}})+\sum_{l \in \mathcal{L}_{c}} X_{l}(t)\left(\bar{\varrho}_{l}^{*}-\bar{\varrho}_{l}^{*}\right)\right] \\
& -\delta^{-1} \sum_{l \in \mathcal{L}_{t}} \sum_{k=1}^{F} \bar{\varrho}_{l, k}^{*} f_{k} U_{l}\left(\frac{1}{f_{k}}\right)-\delta^{-1} \sum_{l \in \mathcal{L}_{c}} U_{l}\left(\bar{\varrho}_{l}^{*}\right),
\end{aligned}
$$

where we have used the notation $I_{l, n}^{S T A T}(t)$ to denote the schedule used by policy $\Upsilon^{S T A T}$, and used the notations $D_{l}^{S T A T}(t)$, $C_{\Omega_{j}, i}^{S T A T}(t)$, and $P_{\Phi_{m}}^{S T A T}(t)$ to denote the resulting transmission rate, interference power, and transmit power, respectively.

It is not hard to show that [18]

$$
\begin{aligned}
& \mathbb{E}\left[Q_{l}(t)\left(\bar{\varrho}_{l}^{*}-D_{l}^{S T A T}(t)\right)\right] \leq \epsilon_{t} Q_{\max } R_{\max }, \\
& \mathbb{E}\left[Y_{j, i}(t)\left(C_{\Omega_{j}, i}^{S T A T}(t)-\hat{C}_{\Omega_{j}, i} s_{\Omega_{j}}^{(p)}(t)\right)\right] \\
& \quad \leq \epsilon_{t} Y_{\max }\left(C_{\max }+\hat{C}_{\max }\right), \\
& \mathbb{E}\left[Z_{m}(t)\left(P_{\Phi_{m}}^{S T A T}(t)-\hat{P}_{\Phi_{m}}\right)\right] \leq \epsilon_{t} Z_{\max } P_{\max },
\end{aligned}
$$

where $\epsilon_{t}$ is a parameter such that $\lim _{t \rightarrow \infty} \epsilon_{t}=0$. Using the inequalities (29)-(32), we have that

$$
\Delta(L(t))-\delta^{-1} \mathbb{E}[g(t)] \leq B+B_{1} \epsilon_{t}-\delta^{-1} g^{*},
$$

where $B_{1}=L Q_{\max } R_{\max }+N_{p}|\Omega|_{\max } Y_{\max }\left(C_{\max }+\hat{C}_{\max }\right)+$ $L Z_{\max } P_{\max }$, with $|\Omega|_{\max }=\max _{1 \leq j \leq N_{\Omega}}\left|\Omega_{j}\right|$, and $g^{*}$ is defined in (22).

The above inequality has the exact form required to apply Proposition 1. Applying the proposition, we obtain

$$
\begin{aligned}
& \liminf _{T \rightarrow \infty}\left\{\sum_{l \in \mathcal{L}_{t}} \sum_{k=1}^{F}\left(\frac{1}{T} \sum_{t=0}^{T-1} \mathbb{E}\left[\varrho_{l, k}(t)\right]\right) f_{k} U_{l}\left(\frac{1}{f_{k}}\right)\right. \\
& \left.+\sum_{l \in \mathcal{L}_{c}} \mathbb{E}\left[\frac{1}{T} \sum_{t=0}^{T-1} U_{l}\left(\xi_{l}(t)\right)\right]\right\} \geq g^{*}-\delta B .
\end{aligned}
$$

On the other hand, we have that

$$
\begin{aligned}
\mathbb{E}\left[\frac{1}{T} \sum_{t=0}^{T-1} U_{l}\left(\xi_{l}(t)\right)\right] & \leq \mathbb{E}\left[U_{l}\left(\frac{1}{T} \sum_{t=0}^{T-1} \xi_{l}(t)\right)\right] \\
& \leq \mathbb{E}\left[U_{l}\left(\frac{1}{T} \sum_{t=0}^{T-1} \varrho_{l}(t)+\frac{X_{\max }}{T}\right)\right],
\end{aligned}
$$

where the first inequality follows from the concavity of $U_{l}(\cdot)$. The second inequality can be obtained by noting that $U_{l}(\cdot)$ is non-decreasing and that $\Upsilon^{A C S}$ keeps control queues deter- 
ministically bounded such that $X_{l}(t) \leq X_{\max }$, and hence, we should have $\sum_{t=0}^{T-1} \xi_{l}(t)-\sum_{t=0}^{T-1} \varrho_{l}(t) \leq X_{\max }$. Using (34) and (35) (for large $T$ ), we obtain the performance bound in the theorem, as required.

\section{Complexity Reduction AND Distributed IMPLEMENTATION}

The scheduling problem in (20) is in general a complex problem and hard to solve. In order to understand the performance of ACS under practical implementation, we next study the application of sub-optimal scheduling policies.

Considering that SAPs naturally operate autonomously, we consider a general model amenable to distributed implementation. Suppose SAPs are able to find a schedule vector that only with probability $\alpha$ ensures a $\beta$ fraction of the optimal objective function in (20). Let this imperfect scheduling policy be $\Upsilon_{s}^{I M P}$. We have the following theorem, whose proof is provided in [18].

Theorem 2. Consider policy $\Upsilon^{I M P}$ that uses the imperfect scheduling policy $\Upsilon_{s}^{I M P}$ for scheduling and admission control of Section V-B.1. $\Upsilon^{I M P}$ guarantees the following performance bound:

$$
\begin{aligned}
\liminf _{T \rightarrow \infty}\{ & \sum_{l \in \mathcal{L}_{t}} \sum_{k=1}^{F}\left(\frac{1}{T} \sum_{t=0}^{T-1} \mathbb{E}\left[\varrho_{l, k}^{I M P}(t)\right]\right) f_{k} U_{l}\left(\frac{1}{f_{k}}\right) \\
& \left.+\sum_{l \in \mathcal{L}_{c}} \mathbb{E}\left[U_{l}\left(\frac{1}{T} \sum_{t=0}^{T-1} \varrho_{l}^{I M P}(t)\right)\right]\right\} \geq \alpha \beta g^{*}-\delta B .
\end{aligned}
$$

This theorem states that using an imperfect scheduling policy as described, we can still achieve an average performance that is a constant-factor of the optimal solution. It is an immediate extension to show that all queue bounds hold as stated in Theorem 1.

One example for $\Upsilon_{s}^{I M P}$ is the Generalized Greedy Maximal Weighted Independent Set (GGMWIS) algorithm [17], which can be used as follows. For each band $n$, perform the following. Consider the interference graph $G(n)$ induced by band $n$ and parameters $\gamma_{l_{1}, n}^{l_{2}}$,s. Select MN $l$ that satisfies $w_{l, n}(t) \geq \sum_{l_{1} \in N_{G(n)}^{+}(l)} w_{l_{1}, n}(t) /\left[d_{G}\left(l_{1}\right)+1\right]$, where $N_{G(n)}^{+}(l)$ is the neighborhood of $l$ in $G(n)$ plus $l$, and $d_{G(n)}\left(l_{1}\right)$ is the degree of MN $l_{1}$ in $G(n)$. Set $I_{l, n}(t)=1$ for the selected MN. Update $G(n)$ by removing $N_{G(n)}^{+}(l)$, which includes $\mathrm{MN} l$ and its interfering MNs, from $G(n)$. Repeat the same process for the updated $G(n)$ and stop when no MN is left for scheduling in band $n$. Note that the GGMWIS algorithm mainly uses neighborhood information in the interference graphs, which renders this algorithm suitable for distributed implementation. For the GGMWIS algorithm, $\alpha=1$ and $\beta=\frac{1}{\Delta}$ [17], where $\Delta$ is the maximum graph-degree over all given $G(n)$ 's. Therefore, applying Theorem 2, we see that for a given network, the simple GGMWIS algorithm provides a performance that is arbitrarily close to $\frac{1}{\Delta}$ of the optimal.

\section{CONCLUSION}

We have developed an admission control and scheduling policy for a heterogenous cognitive radio network with dynamic user arrivals and departures. The policy achieves throughput utility performance arbitrarily close to optimal, while respecting a set of constraints on the received interference and average transmit power. Our analysis further quantifies the tradeoff between the policy's throughput utility performance and the average delay in admitting users. The proposed policy uses several control virtual queues and provides deterministic bounds on the excess received interference on primary transmissions and excess transmit power of secondary users. Due to the structure of interference constraints, the policy can be complex in general. Therefore, we have further considered a class of imperfect policies and shown that these policies are constantfactor approximations of the optimal policy.

\section{REFERENCES}

[1] Q. Zhao and B. Sadler, "A survey of dynamic spectrum access," IEEE Signal Process. Mag., vol. 24, no. 3, May 2007.

[2] I. Akyildiz, W.-Y. Lee, M. Vuran, and S. Mohanty, "A survey on spectrum management in cognitive radio networks [cognitive radio communications and networks]," IEEE Commun. Mag., vol. 46, no. 4, Apr. 2008.

[3] L. Cao and H. Zheng, "Stable and efficient spectrum access in next generation dynamic spectrum networks," in Proc. IEEE INFOCOM'08, Apr. 2008.

[4] M. Neely, E. Modiano, and C. Rohrs, "Dynamic power allocation and routing for time-varying wireless networks," IEEE J. Sel. Areas Commun., vol. 23, no. 1, pp. 89-103, Jan. 2005.

[5] A. Sabharwal and A. K. E. Knightly, "Opportunistic spectral usage: Bounds and a multi-band CSMA/CA protocol," IEEE/ACM Trans. Netw., vol. 15, no. 3, June 2007.

[6] N. Chang and M. Liu, "Competitive analysis of opportunistic spectrum access strategies," in Proc. IEEE INFOCOM'08, Apr. 2008.

[7] D. Niyato and E. Hossain, "Competitive pricing for spectrum sharing in cognitive radio networks: Dynamic game, inefficiency of nash equilibrium, and collusion," IEEE J. Sel. Areas Commun., vol. 26, no. 1, Jan. 2008.

[8] Y. Wu and D. Tsang, "Distributed power allocation algorithm for spectrum sharing cognitive radio networks with QoS guarantee," in Proc. IEEE INFOCOM'09, April 2009.

[9] J. Bae, E. Beigman, R. Berry, M. Honig, and R. Vohra, "Efficiency of sequential bandwidth and power auctions with rate utilities," in 2nd International Conference on Cognitive Radio Oriented Wireless Networks and Communications (CrownCom), Aug. 2007.

[10] Y. Hou, Y. Shi, and H. Sherali, "Optimal spectrum sharing for multi-hop software defined radio networks," in Proc. IEEE INFOCOM'07, May 2007.

[11] Y. Chen, Q. Zhao, and A. Swami, "Joint design and separation principle for opportunistic spectrum access in the presence of sensing errors," IEEE Trans. Inf. Theory, vol. 54, no. 5, May 2008.

[12] S. Huang, X. Liu, and Z. Ding, "Opportunistic spectrum access in cognitive radio networks," in Proc. IEEE INFOCOM'08, Apr. 2008.

[13] R. Urgaonkar and M. Neely, "Opportunistic scheduling with reliability guarantees in cognitive radio networks," in Proc. IEEE INFOCOM'08, Apr. 2008.

[14] L. Cao and H. Zheng, "Distributed spectrum allocation via local bargaining," in Proc. IEEE SECON'05, Sep. 2005.

[15] C. Peng, H. Zheng, and B. Zhao, "Utilization and fairness in spectrum assignment for opportunistic spectrum access," ACM Mobile Networks and Applications Journal, vol. 11, no. 4, 2006.

[16] M. Neely, "Energy optimal control for time-varying wireless networks," IEEE Trans. Inf. Theory, vol. 52, no. 7, Jul. 2006.

[17] S. Sakai, M. Togasaki, and K. Yamazaki, "A note on greedy algorithms for maximum weighted independent set problem," Discrete Applied Mathematics, vol. 126, pp. 313-322, 2003.

[18] M. Lotfinezhad, B. Liang., and E. Sousa, "Optimal control of constrained cognitive radio networks with dynamic population size," University of Toronto, Tech. Rep. [Online]. Available: http://www.comm.utoronto.ca/ liang/publications/preprints/ACS.pdf

[19] L. Tassiulas and A. Ephremides, "Stability properties of constrained queueing systems and scheduling policies for maximum throughput in multihop radio networks," IEEE Trans. Autom. Control, vol. 37, no. 12, pp. 1936-1948, Dec. 1992. 